\title{
Melatonin mitigates cisplatin-induced acute kidney injury through regulation of the heat shock proteins expressions
}

Ali Tuğrul Akin ( $\square$ tugrul.akinn@gmail.com )

Erciyes Üniversitesi: Erciyes Universitesi https://orcid.org/0000-0002-1408-8571

\section{Murat Unsal}

Erciyes Üniversitesi: Erciyes Universitesi

\section{Tayfun Ceylan}

Kapadokya Üniversitesi: Kapadokya Universitesi

\section{Emin Kaymak}

Yozgat Bozok Üniversitesi: Yozgat Bozok Universitesi

\section{Emel Ozturk}

Harran Üniversitesi: Harran Universitesi

\section{Nurhan Kuloglu}

Nigde Omer Halisdemir Universitesi

\section{Derya Karabulut}

Erciyes Üniversitesi: Erciyes Universitesi

\section{Birkan Yakan}

Erciyes Üniversitesi: Erciyes Universitesi

\section{Research Article}

Keywords: Cisplatin, melatonin, heat-shock proteins, acute kidney injury

Posted Date: July 13th, 2021

DOI: https://doi.org/10.21203/rs.3.rs-703570/v1

License: (1) This work is licensed under a Creative Commons Attribution 4.0 International License. Read Full License 


\section{Abstract \\ Purpose.}

To determine the protective effects of melatonin (MEL) in acute kidney injury (AKI) induced by Cisplatin (CP), widely used as a chemotherapeutic in the treatment of many cancer types, via assessment of heatshock proteins (HSPs) levels in rats.

\section{Methods.}

Total 40 Wistar albino rats were divided into four groups: Control $(n=10)$, MEL $(n=10,10 \mathrm{mg} / \mathrm{kg} / \mathrm{i} . \mathrm{p}$ melatonin for 8 days), CP ( $n=10,7 \mathrm{mg} / \mathrm{kg} / \mathrm{i} . \mathrm{p}$ cisplatin at the 5 th day), and CP + MEL $(n=10,10$ $\mathrm{mg} / \mathrm{kg} / \mathrm{i}$.p melatonin for 8 days and $7 \mathrm{mg} / \mathrm{kg} / \mathrm{i} . \mathrm{p}$ cisplatin at the 5 th day). After administrations, animals were sacrificed, and kidney tissues were extracted. Histopathological changes were evaluated and glomerular and tubular immunoreactivities of HSP47, HSP60, HSP7, and HSP90 in renal cortex were detected by immunohistochemistry. Moreover, blood serum BUN, creatinine and uric acid levels were measured to assess of kidney function.

\section{Results.}

CP group showed histopathological deterioration compared to Control group and MEL treatment attenuated this damage. When compared with Control and MEL groups, an increase in HSPs immunoreactivities in renal cortex and blood serum BUN, creatinine and uric acid levels were observed in the CP group. Furthermore, an improvement was observed in the CP + MEL in terms of these parameters compared to the CP group.

\section{Conclusion.}

According to our results, MEL could exert a significant protective effect to ameliorate CP-induced AKI via regulation of heat-shock protein expressions.

\section{Introduction}

Due to increasing success rate of chemotherapy, the long-term survival ratios of the patients and their life quality have increased nowadays in cancer treatment. However, chemotherapeutics may cause a significant damage in healthy organs because their mechanism of action could not be completely understood (Patel and Kaufmann, 2012). The kidney is one of the organs where chemotherapeutics cause devastating side effects, and nephrotoxicity is one of the most widely known and studied side effects (Lampe et al., 1997). Cisplatin (CP), also known as CP-diaminedichloroplatinum-II, is widely used in the treatment of many types of cancer such as lung, breast, ovarian, testicular, and head and neck 
cancers thanks to its anticarcinogenic properties (Dasari and Tchounwou, 2014, CG, 2018). However, it has many side effects such as neurotoxicity, nephrotoxicity, hepatotoxicity, gonadotoxicity (Lu and Cederbaum, 2006, Melli et al., 2008, Santos et al., 2008, Quintanilha et al., 2017, Kohsaka et al., 2020).

Applications of CP in cancer treatment cause acute kidney injury (AKI) in patients and CP-induced AKI is a complex pathophysiological process associated with apoptosis, vascular damage, oxidative and endoplasmic reticulum stress, and inflammation (McSweeney et al., 2021). Several studies have shown that renal function parameters are severely altered in CP-induced AKI. Blood urea nitrogen (BUN), creatinine and uric acid, whose levels increase in serum due to impaired renal function in CP-induced AKI, are commonly used markers of kidney damage (Parlakpinar et al., 2002).

In various tissues and cells, exposure to stress conditions such as thermal stress induces overexpressions of several stress proteins called heat-shock proteins (HSPs). Inducible HSPs are synthesized in response to several process such as inflammation, ischemia, and oxidative stress as defense molecules that protect tissues from further injury. The activity of HSPs (chaperones) is one of the most effective kidney protection mechanisms. HSPs are divided into several subfamilies based on their molecular weight in kilodaltons $(\mathrm{kDa})$. Many HSPs are expressed in healthy kidney tissue, and their expression levels change in various conditions of injury (Beck et al., 2000).

Heat shock protein (HSP) 47 is a specific chaperone that stabilizes the higher-order structure of procollagen (Tasab et al., 2000). It is likely that increased glomerular expression of the collagen-specific chaperone protein HSP47 is responsible for the excessive production of collagen that ultimately leads to glomerular sclerosis (Ohashi et al., 2004). Thus, HSP47 is thought to have an important function in regulating ECM synthesis and degradation in processes related to renal tubulointerstitial fibrosis (Xiao et al., 2012). The HSP60 family includes molecular chaperones that cross-link monomeric proteins and assemble them into oligomeric complexes. Tubular damage can be delayed by preventing Bax-mediated apoptosis through early stimulation of HSP60 in CP-induced acute kidney injury (Chebotareva et al., 2017). Among the families of HSPs, HSP70 has a wide range of functions common to all chaperone proteins. Shaping the structure of newly synthesized native proteins, recovery of partially denatured proteins and degradation of irreversibly damaged protein molecules and transport of proteins from intracellular membranes to organelles through interaction with the cytoskeleton are among the intracellular functions of HSP70 (Beck et al., 2000). HSP70 suppresses inflammation and apoptosis by inhibiting the phosphorylation of stress kinases (JNK and p38 MAPK), which leads to inhibition of both apoptosis and synthesis of proinflammatory cytokines (Chebotareva et al., 2017). Ubiquitously expressed in healthy cells, the HSP90 complex aids in the folding and function of various proteins such as tyrosine kinases (eg, Akt and MEK), transcription factors (androgen receptor, estrogen receptor, and p53), structural proteins (tubulin, actin), and hypoxia-inducible factor-1alpha (HIF-1a) (Goetz et al., 2003). In ischemia or toxic agents-induced AKI, HSP90 expression is increased in tubular cells. Thus, HSP90 is a component of the protective system that ensures regeneration through the repair of damaged tubules and the differentiation of new tubular cells (Morita et al., 1995). 
Melatonin (MEL) is a hormone that plays a role in regulating the circadian rhythm in the body. MEL regulates the reduction/oxidation system under stress conditions such as heat stress and toxicity and it has many biological properties such as anti-inflammation, antioxidant, and anti-apoptosis (Farhood et al., 2019). Many studies have showed that MEL protects kidney tissue from the side effects of CP by inhibiting oxidative stress, inflammation, and apoptosis in CP-induced AKI.

We know that HSPs are overexpressed when cell function does not maintain regularly and is disrupted by various reasons such as ischemia and toxicity. Therefore, it is inevitable that oxidative stress, inflammation, and apoptosis caused by CP administrations in the kidney tissue will not activate HSPs. After a detailed literature review, it was concluded that studies on the effect of MEL on HSPs expressions in CP-induced AKI are insufficient. Therefore, in this study, we aimed to show the effect of MEL, which has many biological properties, on HSPs expressions in CP-induced AKI. We evaluated kidney tissue damage histologically and compared the expression levels of HSP47, HSP60, HSP70 and HSP90 in kidney tissue. In addition, we demonstrated the effect of MEL on the increased serum BUN, creatinine, and uric acid levels by $\mathrm{CP}$ applications via biochemical methods. This study aimed to demonstrate the therapeutic effects of MEL on HSPs against CP-induced AKI.

\section{Materials And Methods}

\section{Experimental design}

The experimental protocol of this study was accepted by the Erciyes University's Experimental Animal and Local Ethics' Committee with number 21/136/2021. In this study, all the animals received human care according to standard guidelines. Forty male Wistar albino rats (9 weeks old, weighing 200-250 gr) were obtained from Hakan Cetinsaya Experimental and Clinic Research Center, Erciyes University, Kayseri, Turkey. Rats were kept at room temperature $\left(20-24^{\circ} \mathrm{C}\right)$ for 12 hours light / 12 hours dark cycle and ambient humidity for the duration of the experiment. Standard chow and tap water were given to animals ad libitum. Total 40 Wistar albino rats randomly divided into four groups as follows; The Control group $(\mathrm{n}=10)$ were untreated rats, the MEL group $(\mathrm{n}=10)$ administered $10 \mathrm{mg} / \mathrm{kg} / \mathrm{i} . \mathrm{p}$ for 8 days ( $\mathrm{Oz}$ and Ihan, 2006), the CP group $(\mathrm{n}=10)$ given $7 \mathrm{mg} / \mathrm{kg} / \mathrm{i} . \mathrm{p} \mathrm{CP}$ on the 5 th day of the experiment (Kandemir et al., 2019) and the CP+MEL group $(\mathrm{n}=10)$ injected with $7 \mathrm{mg} / \mathrm{kg} / \mathrm{i} . \mathrm{p} \mathrm{CP}$ on the 5 th day of the experiment and MEL for 8 days. Administrations continued for 8 days. After experimental procedure, animals were anesthetized with $30 \mathrm{mg} / \mathrm{kg}$ ketamine and $4 \mathrm{mg} / \mathrm{kg}$ xylazine and they were sacrificed and kidney tissues and blood samples were taken for the histopathological, immunohistochemical and biochemical examinations.

\section{Histopathological evaluation}

After scarification of the animals, the kidney tissues were extracted and were fixed in $4 \%$ formaldehyde for histological examination. Following dehydration and clearing, they were embedded in paraffin. Sections were stained and photographs were taken with a light microscope for histopathological evaluation (Olympus BX51, Center Valley, PA). 
Paraffin sections were incubated at $58^{\circ} \mathrm{C}$ for 2 hours for deparaffinization. After deparaffinization, sections were rehydrated with alcohol series, and they were washed with tap water. At room temperature, the sections stained with Hematoxylin and eosin respectively. After dehydration and clearing, sections were examined with a light microscope to detect the histopathological changes in the kidney tissues of experimental groups.

\section{Kidney injury score}

For histopathological scoring of kidney sections, the following criteria were used; glomerular degeneration, tubular epithelial degeneration, tubular dilatation, hemorrhage and mononuclear cell filtration. Scoring was conducted as follows: $0=$ not at all, $1=0-25 \%, 2=26-45 \%, 3=46-75 \%$, and $4=$ 76-100\% (Ozbilgin et al., 2016). Obtained quantitative data analyzed and compared among experimental groups.

\section{Calculation of Bowman's space percentage}

Bowman's capsule diameter and glomerular capillary tuft diameter were measured by Image $\mathrm{J}$ program (1.45s, National Institute of Health, USA, RRID: SCR_003070) on the photographs. After measuring, Bowman's space percentage was calculated via a formula given below. Changes in the \% Bowman's space area were statistically analyzed and compared among the experimental groups (Kashif et al., 2021).

$$
\% \text { Bowman'space }=\frac{(\mathrm{BC} \text { diameter }- \text { GCT diameter }) \times 100}{\mathrm{BC} \text { diameter }}
$$

- BC: Bowman's capsule

- GCT: Glomerular capillary tuft

\section{Immunohistochemical staining}

Immunohistochemistry method was used to investigate HSP47, HSP60, HSP70, and HSP90 immunoreactivities in the kidney sections. $5 \mu \mathrm{m}$ sections were gotten from kidney tissues embedded in paraffin blocks. The sections were kept in the oven at 60 degrees for at least 2 hours so that the paraffin melted. The tissues were deparaffinized and rehydrated using xylene and alcohol series. Sections were taken into $0.01 \mathrm{M}$ citrate buffer and heated in microwave oven at $350 \mathrm{~W}$, consequently antigen retrieval was obtained. Then, sections were kept three times in phosphate buffered saline (PBS) for 5 minutes. The sections were kept in $3 \%(\mathrm{w} / \mathrm{v}) \mathrm{H}_{2} \mathrm{O}_{2}$ for 10 minutes to block endogenous peroxidase activity. After washing again 3 times in PBS, ultra $v$ block solution was added to the tissues and kept in the tank for 5 minutes. After then, HSP47 (Cat. No: BS-1538R, Bioss, USA), HSP60 (Cat. No: BS-0191R, Bioss, USA), HSP70 (Cat. No: BS-0126R, Bioss, USA) and HSP90 (Cat. No: BS-0135R, Bioss, USA) 
antibodies were added to the tissues and incubated overnight at $4{ }^{\bullet} \mathrm{C}$. The following morning, the tissues were washed again 3 times with PBS and the secondary antibody (TA-125-HDX, Thermo Fisher Scientific, Waltham, MA, USA) was instilled for 10 minutes at room temperature. After washing with PBS, the immunoreaction was amplified using the streptavidin-avidin-peroxidase complex and the sections were visualized using 3,30-p-diaminobenzidine tetrahydrochloride (TA-060-HDX, Thermo Fisher Scientific, Waltham, MA, USA) lightly counter-stained with Gill hematoxylin. For the final step, increasing alcohol serial concentrations were used to remove water, the sections were then passed through xylene, and finally, they were covered with an entellan (Karabulut et al., 2020).

\section{Semi-quantitative immunohistochemistry}

All stained sections from each experimental group were evaluated for the immunoreactivity of HSP47, HSP60, HSP70 and HSP90, and photographs were taken in 32 microscopic fields for each group. The intensity of immunoreactivity in both the cortical tubules and the glomerulus on these 32 photographs was measured with the Image J program (1.45s, National Institute of Health, USA, RRID: SCR_003070). For each experimental group, a total of 64 measurements were taken equally from both the cortical tubules and the glomerulus. A total of 480 measurements from 240 microscopic areas were taken and evaluated for each group. The quantitative data obtained were statistically analyzed and compared among the experimental groups (Akin AT, 2020).

\section{Biochemical analyzes}

Blood samples from experimental animals were centrifuged and serum samples were obtained for further biochemical analysis. Before the examinations of the serum samples, they were kept at $-80^{\circ} \mathrm{C}$. To assess the kidney function, serum blood urea nitrogen (BUN), creatinine and uric acid levels were measured by Roche $^{\text {TM }}$ Cobas 8000 Modular Analyzer device at the Erciyes University Central Biochemistry Laboratory. Obtained measurements were statistically analyzed and compared among the experimental groups.

\section{Statistical Analysis}

All statistical analyses were carried out by using GraphPad Prism version 9.00 for Mac, GraphPad Software, La Jolla, California, USA. D'Agostino Pearson omnibus test and Shapiro-Wilk test were used to identify the normal distribution of the data. Quantitative variables were compared using one-way analysis of variance (ANOVA) and Tukey's post-hoc test. The data were expressed as the mean of normalized data \pm standard deviation of the mean. $p<0.05$ was considered as statistically significant.

\section{Results}

\section{Histopathological findings}

Melatonin reduced cisplatin-induced kidney injury 
H\&E staining were performed to investigate the histopathological changes in the kidney tissue. Normal kidney tissue morphology was observed in the Control and MEL groups. In the CP group, glomerular degeneration, tubular epithelial degeneration, tubular dilatation, hemorrhage, and mononuclear cell filtration were observed and a significantly increase was observed in kidney injury score compared to the Control and MEL groups $(p<0.0001)$ (Figure 2A, 2B). Unlikely, the kidney tissue morphology in the CP+ MEL group was similar to the Control and MEL groups. Glomerular degeneration, tubular epithelial degeneration and mononuclear cell filtration were negligible. However, tubular dilatation and hemorrhagic areas were detected in only a few sites (Figure 2A). In terms of these histopathological criteria, there was a statistically significant decrease in the kidney injury score in CP+MEL group compared to CP group $(p<0.0001)$ (Figure 2B). For detailed information about histopathological analyzes, please check the supplementary data.

Improvement of degenerated glomerular morphometry by melatonin

To determine the differences in glomerular morphometry among the experimental groups, the \%Bowman's space was calculated by using the formula given in the materials and methods section (Table 1). As a result of the statistical evaluation of the \%Bowman's spaces, it was determined that the \%Bowman's space was significantly increased in the CP group compared to the Control and MEL groups, indicating that CP significantly reduced the glomerular capillary tufts by causing glomerular degeneration in the kidney tissue. However, the \%Bowman's space in the CP+MEL group was similar to that of the Control and MEL groups. This indicates that melatonin has an ameliorative effect on glomerular degeneration (Figure 2C).

\section{Melatonin reduces the increased heat-shock protein expressions}

Immunohistochemical staining was performed by using the avidin-biotin method to determine the kidney tissue expressions of HSP47, HSP60, HSP70 and HSP90. Immunohistochemical stainings and statistical analysis of the immunoreactivity intensity measurements demonstrated the presence of HSP47, HSP60, HSP70 and HSP90 immunoreactivity in the kidney tissue of all experimental groups. The HSP47, HSP60, HSP70 and HSP90 expressions in the kidney tissues of MEL group were similar to those in the Control group. However, immunoreactivity of these factors significantly increased in kidney tissue of the experimental animals in the CP group. In addition, HSP47, HSP60, HSP70 and HSP90 expressions in the $\mathrm{CP}+\mathrm{MEL}$ group were substantially less compared to those in the CP group. Figure 3 and Figure 4 show the immunohistochemical staining of these factors and Figure 5 shows statistical analysis of the immunoreactivity measurements among experimental groups. Detailed immunoblot data can be found in the supplemental material.

\section{Impaired kidney function was recovered by melatonin}

The levels and statistical analyzes of kidney function parameters measured by biochemical assays in blood serum are shown in Figure 6. Serum BUN, creatinine and uric acid levels in the Control and MEL groups were quite similar and there was no statistically significant difference between these groups 
$(p>0.05)$. However, the levels of these parameters in the CP group showed a significant increase compared to the Control and MEL groups $(p<0.05)$. In addition, serum levels of these parameters were lower in the $\mathrm{CP}+\mathrm{MEL}$ group than in the $\mathrm{CP}$ group, showing the mitigative effects of melatonin on impaired renal function $(p<0.05)$. For the biochemical measurements data, see the supplemental material about our biochemical analyzes.

\section{Discussion}

Drug-induced AKI is one of the most common health complications in the world (Mehta et al., 2007). For this reason, many researchers are working on the search for new strategies to alleviate AKI. In addition to focusing on drug release and exploring safer therapeutics, identifying combination strategies of nephrotoxic drugs and therapeutics is considered the ideal treatment for AKI caused by nephrotoxic agents, such as CP, an antineoplastic chemotherapeutic (Ali and Al Moundhri, 2006). Many studies have reported that $\mathrm{CP}$ administrations cause $\mathrm{AKI}$ in the experimental animal models by inducing tubular epithelial injury, changes in glomerular space and infiltration of the inflammatory cells (Abd El-Kader and Taha, 2020, Sadeghi et al., 2020). In this study, we aimed to investigate the potential therapeutic effects of MEL on CP-induced AKI via focusing on HSP expressions. According to our histopathological examination, we observed that $\mathrm{CP}$ induced a serious kidney damage by causing glomerular degeneration, tubular epithelial degeneration, tubular dilatation, hemorrhage, and mononuclear cell filtration. However, in the CP + MEL group, kidney tissues showed a significant improvement. Therefore, we determined that MEL had a serious protective effect against the harmful effects of CP in kidney tissue at a dose of 10 $\mathrm{mg} / \mathrm{kg}$. We think that this ameliorative effects of MEL in kidney tissue are due to its widely known inhibitory effects oxidative stress, inflammation, and apoptosis, and thus protects the kidney tissue from the harmful effects of $\mathrm{CP}$.

HSP47 plays an important role in providing functional conformation of procollagen as well as preventing secretion of abnormal conformation procollagen under stress conditions (Hamilton and Heikkila, 2006). It has been shown in many studies that the expression of HSP47 in the kidney is increased under various toxicity stress conditions (Li et al., 2018). In our study, it was observed that HSP47 expressions increased in both glomeruli and tubules in the renal cortex of experimental animals in the CP group. We think that this increase in HSP47 expressions in the renal cortex is due to glomerular sclerosis and tubulointerstitial fibrosis induced by CP applications. On the other hand, we showed that MEL, which is used as a protective agent against CP-induced AKI, exerted a serious protective effect by preventing glomerular sclerosis and tubulointerstitial fibrosis in the renal cortex at a dose of $10 \mathrm{mg} / \mathrm{kg}$. Therefore, we suggest that the reason for the significantly lower HSP47 expressions in the CP + MEL group compared to the CP group is due to this protective effect of MEL on the renal cortex.

HSP60 is a molecular chaperone with many intracellular functions such as folding of newly synthesized natural proteins, refolding of damaged protein molecules, preventing Bax-mediated apoptosis, promoting translocation of protein to mitochondria, mediating anti-inflammatory response (Chebotareva et al., 2017). Many studies have reported that HSP60 expressions are significantly increased in CP-induced AKI 
(Dogukan et al., 2011). In our study, we suggest that the significantly increased HSP60 expressions in both the glomerulus and tubules in the renal cortex of experimental animals in the CP group compared to the Control and MEL groups are caused by the defects in protein conformations because of the oxidative damage caused by CP in the cells. As a result, we think that cells increase their HSP60 expression as a mechanism of protection against oxidative stress induced by CP. In addition, since CP induces oxidative stress-induced inflammation and apoptosis in kidney tissue, we can say that the effects of HSP60 on Bax-dependent apoptosis and suppression of inflammation also play a role in this process, and therefore, it increases the HSP60 expression of glomerular and tubular cells in the kidney tissue. On the other hand, HSP60 expressions were significantly lower in the group administered MEL at a dose of $10 \mathrm{mg} / \mathrm{kg}$ compared to the CP group. We think that the reason for this is that MEL protects kidney tissue from the harmful effects of CP by causing inhibition of oxidative stress due to its antioxidant properties.

HSP70 is a chaperone proteins and it has several function in the kidney tissue such as shaping the structure of newly synthesized native proteins, recovery of partially denatured proteins and degradation of irreversibly damaged protein molecules (Zhipeng et al., 2006). Moreover, HSP70 suppresses inflammation and apoptosis by inhibiting the phosphorylation of stress kinases (JNK and p38 MAPK) (Chebotareva et al., 2017). Several studies have showed that HSP70 is upregulated in CP-induced AKI and is related to cell protection and survival (Tuzcu et al., 2010). In this study, the expression levels of HSP70 in glomerulus and tubules in the renal cortex were investigated in CP-induced AKI. In line with the data obtained from immunohistochemical staining, we think that the significantly increased HSP70 expressions in the kidney tissues of experimental animals administered CP indicate a serious response to $\mathrm{CP}$ in the kidney tissue. Therefore, we suggest that the increased expression of HSP70 in the renal cortex in the CP treated group occurred to eliminate misfolded proteins because of oxidative damage caused by $\mathrm{CP}$ and to ensure the proper conformation of newly synthesized proteins. In addition, since the inhibitory effect of HSP70 on inflammation and apoptosis is known, we can say that the cells in the kidney tissue try to protect themselves by suppressing inflammation and apoptosis caused by oxidative stress induced by $\mathrm{CP}$. On the other hand, MEL, which was used as a protective agent in this study, showed a serious protective effect on kidney tissue at a dose of $10 \mathrm{mg} / \mathrm{kg}$, causing HSP70 levels in the glomerulus and tubules in the renal cortex to be like those in the Control and MEL groups. We think that this situation occurred because of the inhibitor effects of MEL on protein misfolding and oxidative stress-induced inflammation and apoptosis due to its protective feature in kidney tissue.

As one of the heat-shock proteins which plays a crucial role to ensure proper folding and conformational maturation of proteins, HSP90 is known to be an abundant and ubiquitous molecular chaperone in the body (Miao et al., 2008). Recent studies have reported that HSP90 expression is increased in toxic conditions associated with inflammation, oxidative stress, and apoptosis, such as CP-induced AKI or doxorubicin-induced podocyte injury (Tsuji et al., 2009, Dong et al., 2020). In the presented study, we propose that the increase in the expression of HSP90 in the glomerulus and tubules in the renal cortex of experimental animals administered with $\mathrm{CP}$ is due to protein misfolding because of CP-induced oxidative stress. We also suggest that because of these protein misfoldings due to oxidative stress, cells increase their HSP90 expression in kidney tissue to ensure translation accuracy. Furthermore, we determined that 
MEL has a protective effect against CP applications in kidney tissue, and considering the decrease in HSP90 expressions, this therapeutic agent also has effects on the elimination of protein misfoldings resulting from $\mathrm{CP}$-induced oxidative stress. These results indicate that MEL has positive effects on translation accuracy against the detrimental effects of CP-induced AKI.

Among kidney function parameters, BUN, creatinine, and uric acid are commonly used markers of kidney damage. In many studies, it has been reported that the serum levels of these parameters are significantly increased in CP-induced AKI. In this study, the significant increase in serum BUN, creatinine and uric acid levels in the CP group compared to the Control and MEL groups indicates that CP causes serious damage to the kidney tissue and impairs kidney functions. Moreover, we think that serum BUN, creatinine, and uric acid levels in the CP + MEL group were like those in the Control and MEL groups, since MEL showed a severe protective effect in CP-induced $\mathrm{AKI}$ at a dose of $10 \mathrm{mg} / \mathrm{kg}$, indicating its protective effects on kidney tissue against the harmful effects of CP. As a matter of fact, our histological and immunohistochemical data also support our suggestion.

As a result, CP caused histopathological changes in the kidney tissues of the experimental animals used in this study. The significant increase in the expression of HSP47, HSP60, HSP70 and HSP90 in the glomeruli and tubules in the renal cortex indicates the cellular response to $\mathrm{CP}$. We think that this increase in HSPs expression is due to the increase in the amount of misfolded protein in the renal cortex because of CP-induced glomerular sclerosis, tubular fibrosis, and oxidative stress. In addition, we think that inflammation and apoptosis are induced in kidney tissue with the deterioration of cellular homeostasis because of misfolded protein accumulation. As a matter of fact, the significant increase in the expression of HSP60, HSP70 and HSP90 in the renal cortex, which play a role in the suppression of inflammation and apoptosis in the CP administered group, supports this idea. In this study, it has been proven that 10 $\mathrm{mg} / \mathrm{kg}$ MEL administration is a chemical agent that affects HSPs and kidney function markers in kidney tissue. According to our immunohistochemical and biochemical results, MEL prevented the overexpression of HSPs by forming a protective effect in kidney tissue, and inflammation caused the inhibition of several processes that would result in apoptosis. In line with our results, the protective effects of MEL against the negative effects of CP in AKI induced by CP at a dose of $10 \mathrm{mg} / \mathrm{kg}$ should not be ignored.

\section{Declarations}

\section{Ethics approval}

The experimental protocol performed in the present study was approved by the Erciyes University's Experimental Animal and Local Ethics' Committee with number 21/136/2021.

\section{Consent to participate}

Informed consent was obtained from all individual participants included in the study. 


\section{Consent to publish}

The participant has consented to the submission of the case report to the journal.

\section{Authors' contributions}

The authors declare that all data were generated in-house and that no paper mill was used. ATA, EK, DK and BY designed the study and ATA, EK, and EO and TC performed experiment. ATA, EK, EO and DK contributed to analyze the data. ATA performed the histological analyses. ATA wrote the manuscript. ATA, DK and $B Y$ revised the final version of the manuscript.

\section{Funding}

The author(s) disclosed receipt of the following financial support for the research, authorship, and/or publication of this article: This study has been supported by a grant from the research and technology department of Erciyes University with grant number TTU-2019-9365.

\section{Conflicting interests}

The author (s) declare no conflict of interest at the publication of this article.

\section{Availability of data and material}

The datasets generated during and/or analyzed during the current study are not publicly available due to privacy but are available from the corresponding author on reasonable request.

\section{References}

Abd El-Kader M, Taha RI (2020) Comparative nephroprotective effects of curcumin and etoricoxib against cisplatin-induced acute kidney injury in rats. Acta Histochem: 151534

Akin AT KE, Karabulut D, Doğanyiğit Z, Ceylan T, Toluk A, Özdamar S. (2020) Comparison of the acute and cumulative dose administrations in doxorubicin-induced hepatotoxicity via evaluation of the histopathological changes and Inflammation in rats. Experimental and Applied Medical Science 1: 73-81

Ali BH, Al Moundhri MS (2006) Agents ameliorating or augmenting the nephrotoxicity of cisplatin and other platinum compounds: a review of some recent research. Food Chem Toxicol 44: 1173-1183

Beck FX, Neuhofer W, Müller E (2000) Molecular chaperones in the kidney: distribution, putative roles, and regulation. Am J Physiol Renal Physiol 279: F203-215

CG HMaH (2018) Anticancer metallodrugs: where is the next cisplatin? Future Med Chem 10(6): 615-617

Chebotareva N, Bobkova I, Shilov E (2017) Heat shock proteins and kidney disease: perspectives of HSP therapy. Cell Stress Chaperones 22: 319-343 
Dasari S, Tchounwou PB (2014) Cisplatin in cancer therapy: molecular mechanisms of action. Eur J Pharmacol 740: 364-378

Dogukan A, Tuzcu M, Agca CA, Gencoglu H, Sahin N, Onderci M, Ozercan IH, Ilhan N, Kucuk O, Sahin K (2011) A tomato lycopene complex protects the kidney from cisplatin-induced injury via affecting oxidative stress as well as Bax, Bcl-2, and HSPs expression. Nutr Cancer 63: 427-434

Dong J, Jiang Z, Ma G (2020) Hsp90 inhibition aggravates adriamycin-induced podocyte injury through intrinsic apoptosis pathway. Exp Cell Res 390: 111928

Farhood B, Goradel NH, Mortezaee K, Khanlarkhani N, Najafi M, Sahebkar A (2019) Melatonin and cancer: From the promotion of genomic stability to use in cancer treatment. J Cell Physiol 234: 5613-5627

Goetz MP, Toft DO, Ames MM, Erlichman C (2003) The Hsp90 chaperone complex as a novel target for cancer therapy. Ann Oncol 14: 1169-1176

Hamilton AM, Heikkila JJ (2006) Examination of the stress-induced expression of the collagen binding heat shock protein, hsp47, in Xenopus laevis cultured cells and embryos. Comp Biochem Physiol A Mol Integr Physiol 143: 133-141

Kandemir FM, Yildirim S, Caglayan C, Kucukler S, Eser G (2019) Protective effects of zingerone on cisplatin-induced nephrotoxicity in female rats. Environ Sci Pollut Res Int 26: 22562-22574

Karabulut D, Ozturk E, Kuloglu N, Akin AT, Kaymak E, Yakan B (2020) Effects of vitamin B12 on methotrexate hepatotoxicity: evaluation of receptor-interacting protein (RIP) kinase. Naunyn Schmiedebergs Arch Pharmacol 393: 2473-2480

Kashif AW, Verma N, Verma S, Boruah D, Sahu R, Kalra S, Malik A (2021) Utility of glomerular morphometry in diagnosing pediatric renal disease. Med J Armed Forces India 77: 194-199

Kohsaka T, Minagawa I, Morimoto M, Yoshida T, Sasanami T, Yoneda Y, Ikegaya N, Sasada H (2020) Efficacy of relaxin for cisplatin-induced testicular dysfunction and epididymal spermatotoxicity. Basic Clin Androl 30: 3

Lampe H, Horwich A, Norman A, Nicholls J, Dearnaley DP (1997) Fertility after chemotherapy for testicular germ cell cancers. J Clin Oncol 15: 239-245

Li PC, Li XN, Du ZH, Wang H, Yu ZR, Li JL (2018) Di (2-ethyl hexyl) phthalate (DEHP)-induced kidney injury in quail (Coturnix japonica) via inhibiting HSF1/HSF3-dependent heat shock response. Chemosphere 209: 981-988

Lu Y, Cederbaum Al (2006) Cisplatin-induced hepatotoxicity is enhanced by elevated expression of cytochrome P450 2E1. Toxicol Sci 89: 515-523 
McSweeney KR, Gadanec LK, Qaradakhi T, Ali BA, Zulli A, Apostolopoulos V (2021) Mechanisms of Cisplatin-Induced Acute Kidney Injury: Pathological Mechanisms, Pharmacological Interventions, and Genetic Mitigations. Cancers (Basel) 13

Mehta RL, Kellum JA, Shah SV, Molitoris BA, Ronco C, Warnock DG, Levin A (2007) Acute Kidney Injury Network: report of an initiative to improve outcomes in acute kidney injury. Crit Care 11: R31

Melli G, Taiana M, Camozzi F, Triolo D, Podini P, Quattrini A, Taroni F, Lauria G (2008) Alpha-lipoic acid prevents mitochondrial damage and neurotoxicity in experimental chemotherapy neuropathy. Exp Neurol 214: $276-284$

Miao RQ, Fontana J, Fulton D, Lin MI, Harrison KD, Sessa WC (2008) Dominant-negative Hsp90 reduces VEGF-stimulated nitric oxide release and migration in endothelial cells. Arterioscler Thromb Vasc Biol 28 : $105-111$

Morita K, Wakui H, Komatsuda A, Ohtani H, Miura AB, Itoh H, Tashima Y (1995) Induction of heat-shock proteins HSP73 and HSP90 in rat kidneys after ischemia. Ren Fail 17: 405-419

Ohashi S, Abe H, Takahashi T, Yamamoto Y, Takeuchi M, Arai H, Nagata K, Kita T, Okamoto H, Yamamoto H, Doi T (2004) Advanced glycation end products increase collagen-specific chaperone protein in mouse diabetic nephropathy. J Biol Chem 279: 19816-19823

Oz E, Ilhan MN (2006) Effects of melatonin in reducing the toxic effects of doxorubicin. Mol Cell Biochem 286: $11-15$

Ozbilgin S, Ozkardesler S, Akan M, Boztas N, Ozbilgin M, Ergur BU, Derici S, Guneli ME, Meseri R (2016) Renal Ischemia/Reperfusion Injury in Diabetic Rats: The Role of Local Ischemic Preconditioning. Biomed Res Int 2016: 8580475

Parlakpinar H, Sahna E, Ozer MK, Ozugurlu F, Vardi N, Acet A (2002) Physiological and pharmacological concentrations of melatonin protect against cisplatin-induced acute renal injury. J Pineal Res 33: 161-166

Patel AG, Kaufmann SH (2012) How does doxorubicin work? Elife 1: e00387

Quintanilha JCF, de Sousa VM, Visacri MB, Amaral LS, Santos RMM, Zambrano T, Salazar LA, Moriel P (2017) Involvement of cytochrome P450 in cisplatin treatment: implications for toxicity. Cancer Chemother Pharmacol 80: 223-233

Sadeghi H, Mansourian M, Panahi Kokhdan E, Salehpour Z, Sadati I, Abbaszadeh-Goudarzi K, Asfaram A, Doustimotlagh AH (2020) Antioxidant and protective effect of Stachys pilifera Benth against nephrotoxicity induced by cisplatin in rats. J Food Biochem: e13190

Santos NA, Bezerra CS, Martins NM, Curti C, Bianchi ML, Santos AC (2008) Hydroxyl radical scavenger ameliorates cisplatin-induced nephrotoxicity by preventing oxidative stress, redox state unbalance, 
impairment of energetic metabolism and apoptosis in rat kidney mitochondria. Cancer Chemother Pharmacol 61: 145-155

Tasab M, Batten MR, Bulleid NJ (2000) Hsp47: a molecular chaperone that interacts with and stabilizes correctly-folded procollagen. Embo j 19: 2204-2211

Tsuji T, Kato A, Yasuda H, Miyaji T, Luo J, Sakao Y, Ito H, Fujigaki Y, Hishida A (2009) The dimethylthiourea-induced attenuation of cisplatin nephrotoxicity is associated with the augmented induction of heat shock proteins. Toxicol Appl Pharmacol 234: 202-208

Tuzcu M, Sahin N, Dogukan A, Aslan A, Gencoglu H, Ilhan N, Kucuk O, Sahin K (2010) Protective role of zinc picolinate on cisplatin-induced nephrotoxicity in rats. J Ren Nutr 20: 398-407

Xiao HB, Liu RH, Ling GH, Xiao L, Xia YC, Liu FY, Li J, Liu YH, Chen QK, Lv JL, Zhan M, Yang SK, Kanwar YS, Sun L (2012) HSP47 regulates ECM accumulation in renal proximal tubular cells induced by TGF- $\beta 1$ through ERK1/2 and JNK MAPK pathways. Am J Physiol Renal Physiol 303: F757-765

Zhipeng W, Li L, Qibing M, Linna L, Yuhua R, Rong Z (2006) Increased expression of heat shock protein (HSP)72 in a human proximal tubular cell line (HK-2) with gentamicin-induced injury. J Toxicol Sci 31: 6170

\section{Tables}

Table 1. Glomerular morphometry measurements of experimental groups.

\begin{tabular}{cccccc}
\hline Groups & Control & MEL & CP & CP +MEL & $p$ \\
\hline $\begin{array}{c}\text { Bowman's } \\
\text { capsule diameter }(\mu)\end{array}$ & $76.6 \pm 7.8$ & $76.61 \pm 5.7$ & $75.33 \pm 7.3$ & $74.2 \pm 8.1$ & $\mathbf{0 . 2 1 9}$ \\
\hline $\begin{array}{c}\text { Glomerular capillary } \\
\text { tuft diameter }(\mu)\end{array}$ & $68.6 \pm 7.8$ & $67.9 \pm 5.5$ & $55.5 \pm 5.6^{*}$ & $65.7 \pm 7.1$ & $\mathbf{0 . 0 0 1}$ \\
\hline \% Bowman's space area & $10.7 \pm 1.7$ & $11.5 \pm 1.4$ & $26.6 \pm 4.8^{*}$ & $11.4 \pm 1.1$ & $\mathbf{0 . 0 0 1}$ \\
\hline
\end{tabular}

All data are expressed as the mean $\pm \mathrm{SD}(\mathrm{n}=10) . p<0.05$ was considered as significant.

* shows significant difference in comparison of CP group and the other experimental groups.

Abbreviations: CP, cisplatin; MEL, melatonin.

\section{Figures}




\section{8 days}
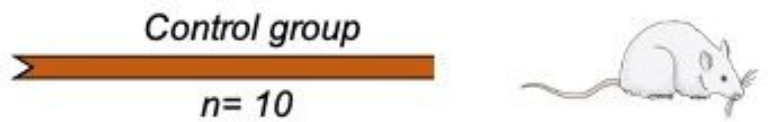

No treatment
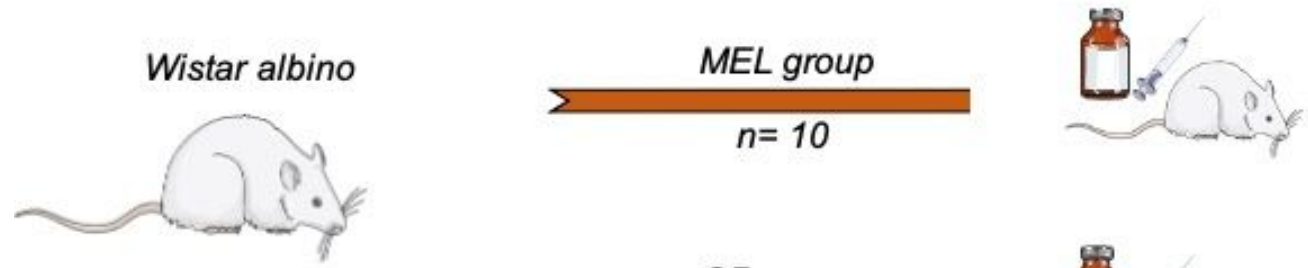

Melatonin (10 mg/kg, i.p)

$n=32$
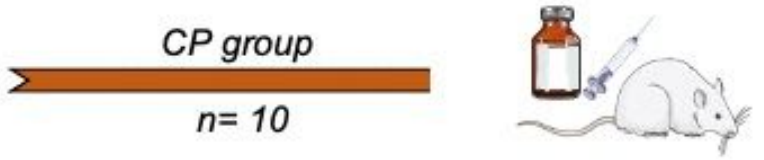

Cisplatin (7 mg/kg, i.p)
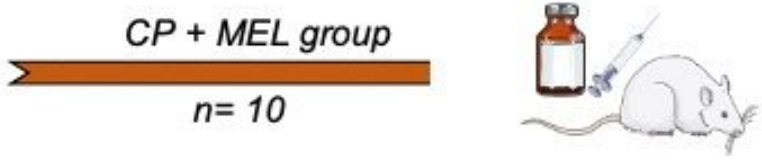

Cisplatin (7 mg/kg, i.p)

Melatonin (10 mg/kg, i.p)

Figure 1

Schematic representation of experimental design.

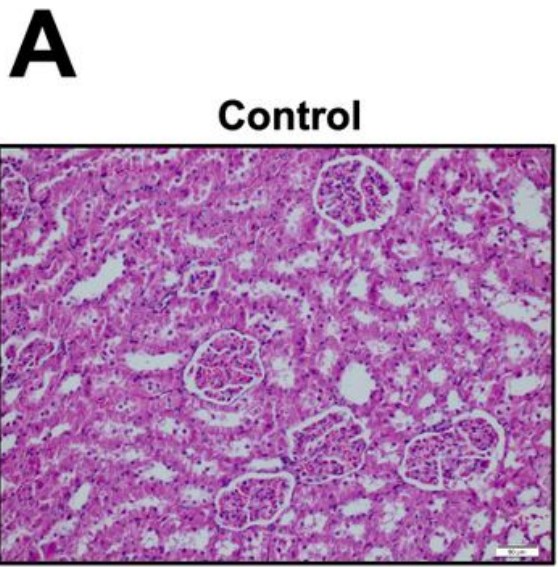

Melatonin (MEL)

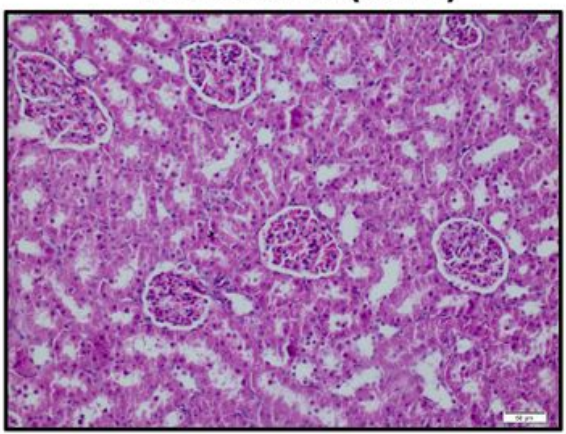

Cisplatin (CP)

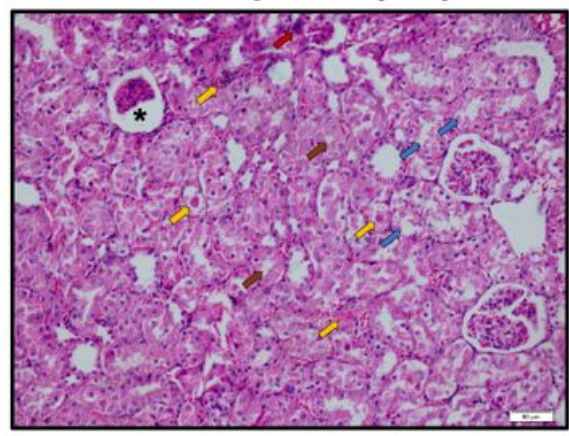

CP + MEL

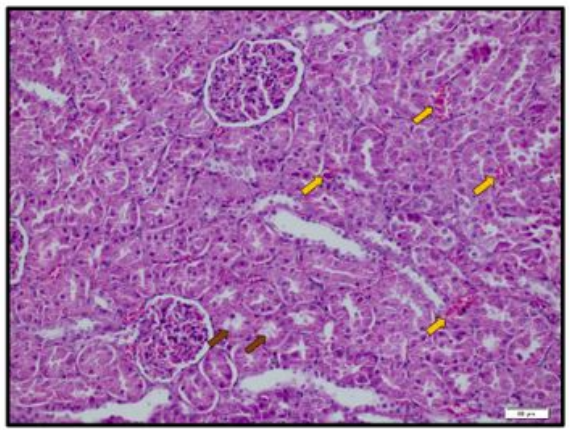

B

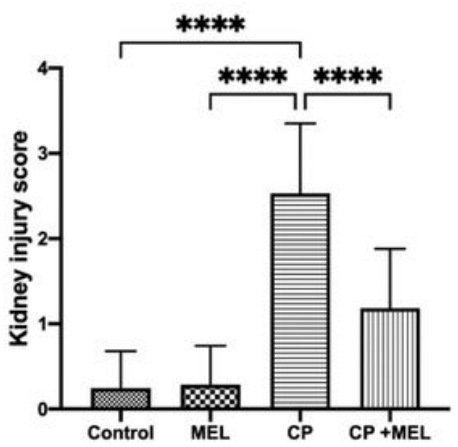

C

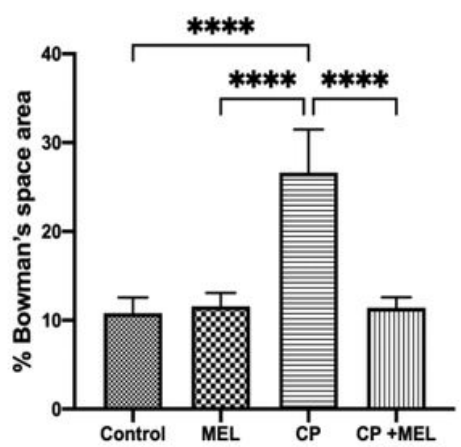


Figure 2

A-C. Light microscopy of kidney tissues stained with H\&E and statistical analysis of kidney injury score and \%Bowman's space area among experimental groups. A H\&E staining of kidney tissues. Control group (untreated rats) and MEL group (given $10 \mathrm{mg} / \mathrm{kg}$ melatonin) showed normal histological structure. CP group (given $7 \mathrm{mg} / \mathrm{kg}$ cisplatin) presented glomerular degeneration (star), tubular epithelial degeneration (blue arrow), tubular dilatation (brown arrow), hemorrhage (yellow arrow) and mononuclear cell filtration (red arrow). $\mathrm{CP}+\mathrm{MEL}$ group showed an improvement in terms of histopathological changes induced by CP. B, C. Statistical analysis of the kidney injury score (B) and \%Bowman's space area (C) among experimental groups. Scale bar $=50 \mu \mathrm{m} .{ }^{*}=p<0.05,{ }^{* *}=p<0.01,{ }^{* * *}=p<0.001, * \star * *=p<0.0001$. Abbreviations: $\mathrm{H} \& \mathrm{E}$, hematoxylin eosin; MEL, melatonin; $\mathrm{CP}$, cisplatin.

\section{Heat-shock protein 47}
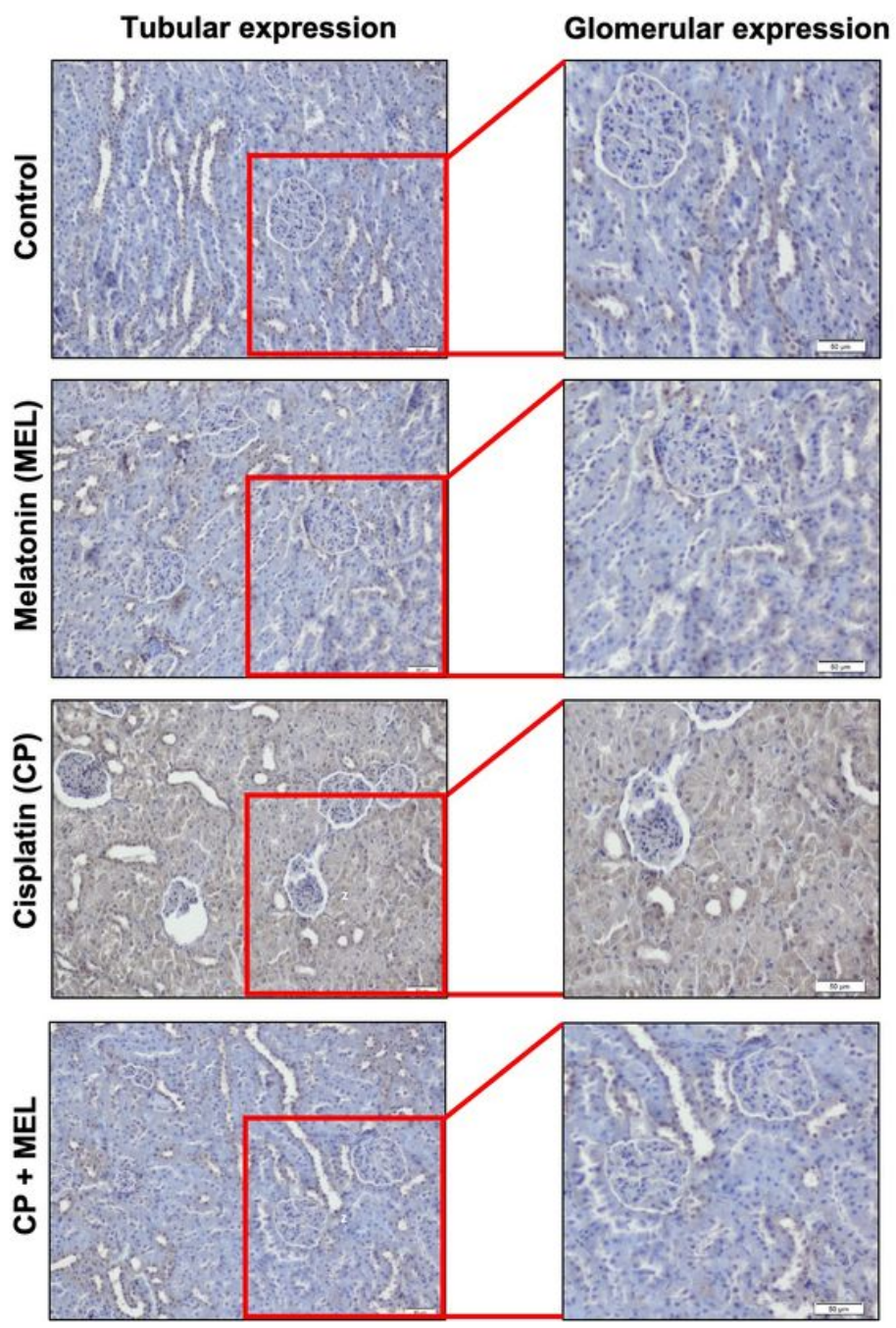

Heat-shock protein 60
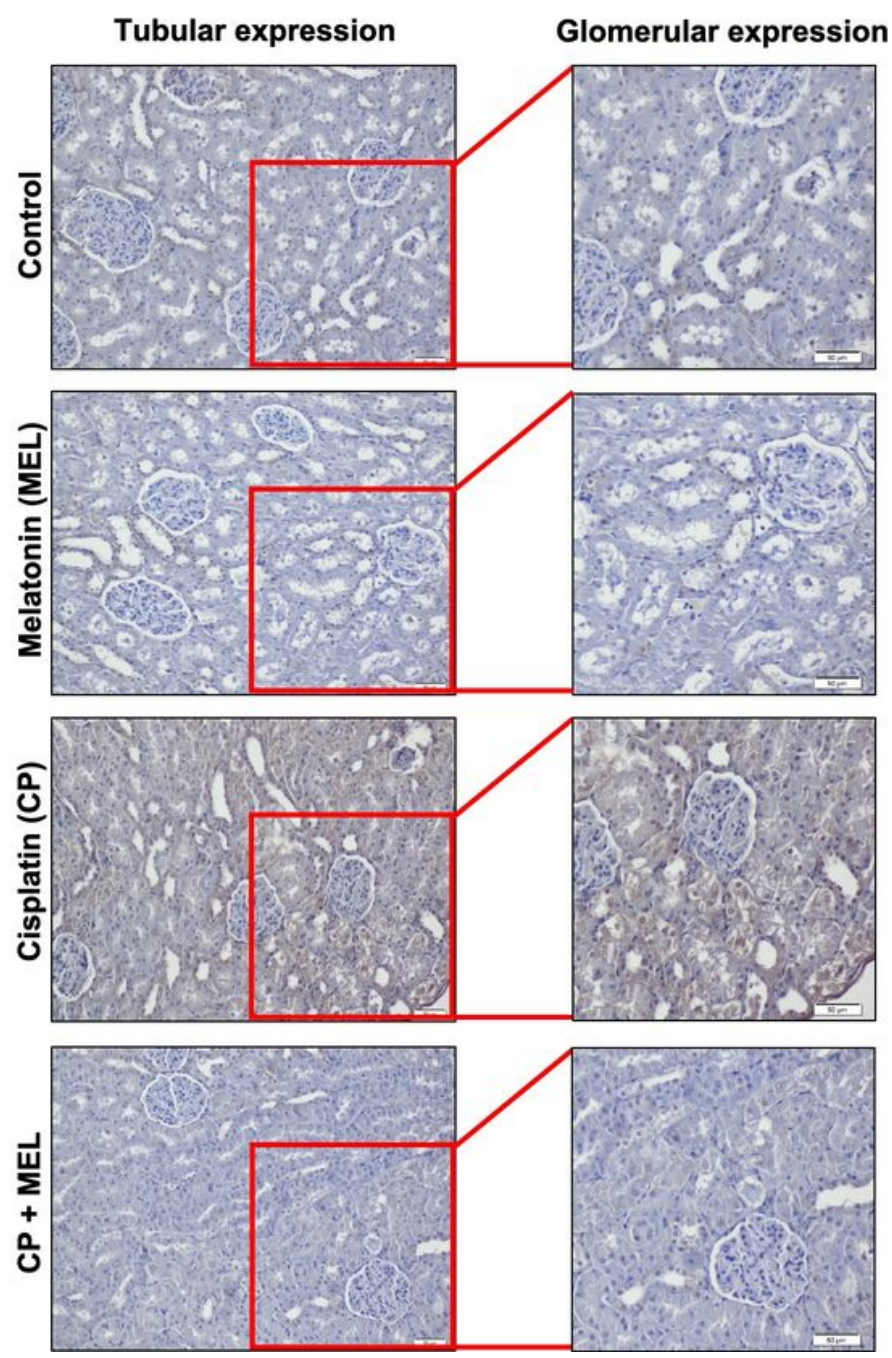

\section{Figure 3}

Immunohistochemical staining of HSP47 and HSP60 in kidney tissues. Control group $(n=10)$ and MEL group $(n=10)$ showed weak HSP47 immunostaining in both glomerular and tubular region of renal cortex in the kidney tissue; CP group ( $n=10)$, HSP47 expressions increased in the glomerular and tubular region. 
$\mathrm{CP}+\mathrm{MEL}$ group $(\mathrm{n}=10)$, HSP47 expressions decreased when compared with CP group. Similarly, weak HSP60 immunostaining was observed in the kidney sections of Control group $(n=10)$ and MEL group $(n=10)$; CP group $(n=10)$, HSP60 expression increased in the glomerular and tubular region; CP+MEL $(n=10)$ HSP60 expressions were less compared to the CP group. Scale bar: $50 \mu \mathrm{m}$. Abbreviations: MEL, melatonin; CP, cisplatin; HSP47, heat-shock protein 47; HSP60, heat-shock protein 60 , HSP70, heat-shock protein 70; HSP90, heat-shock protein 90.

\section{Heat-shock protein 70}
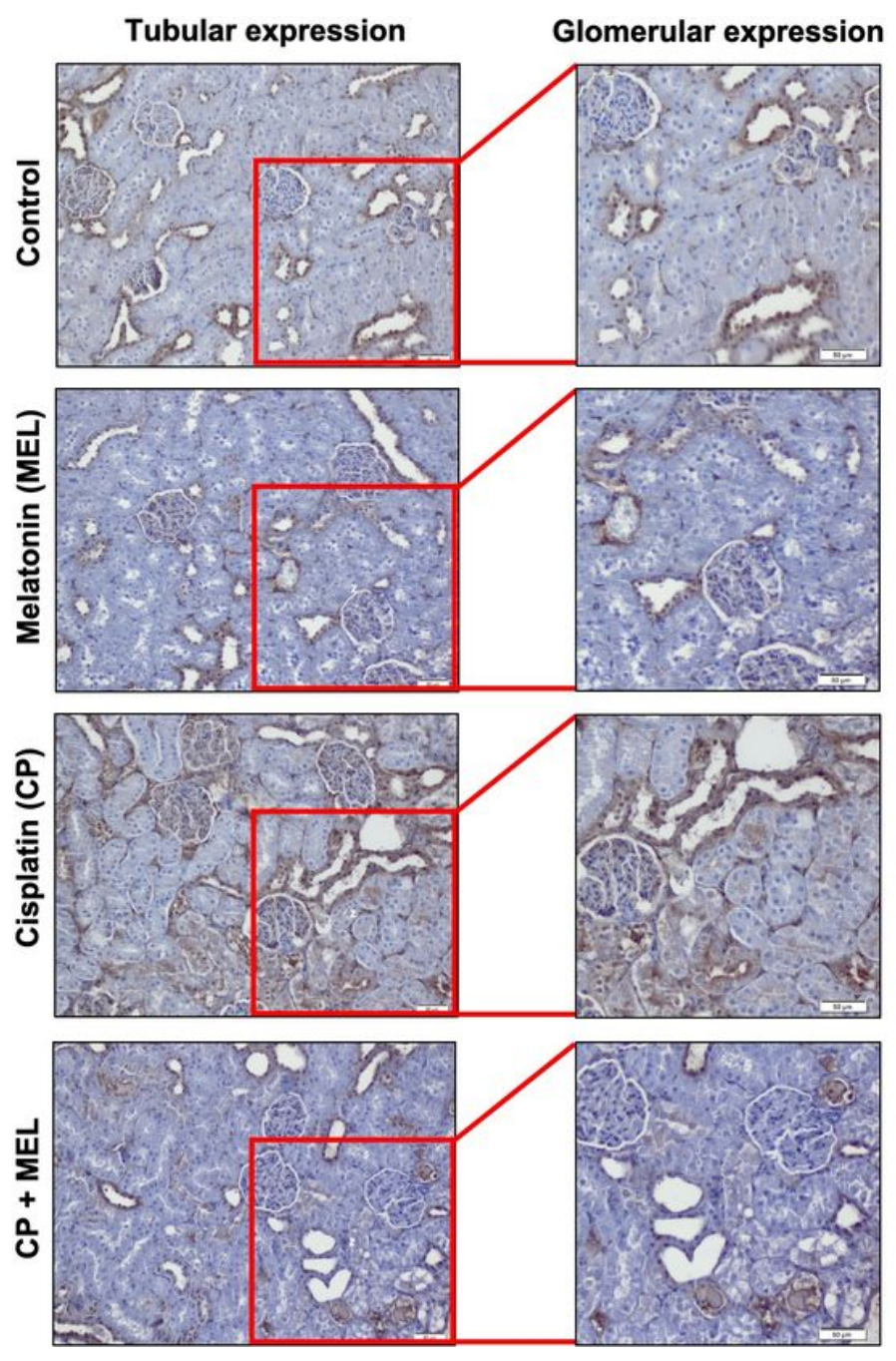

\section{Heat-shock protein 90}
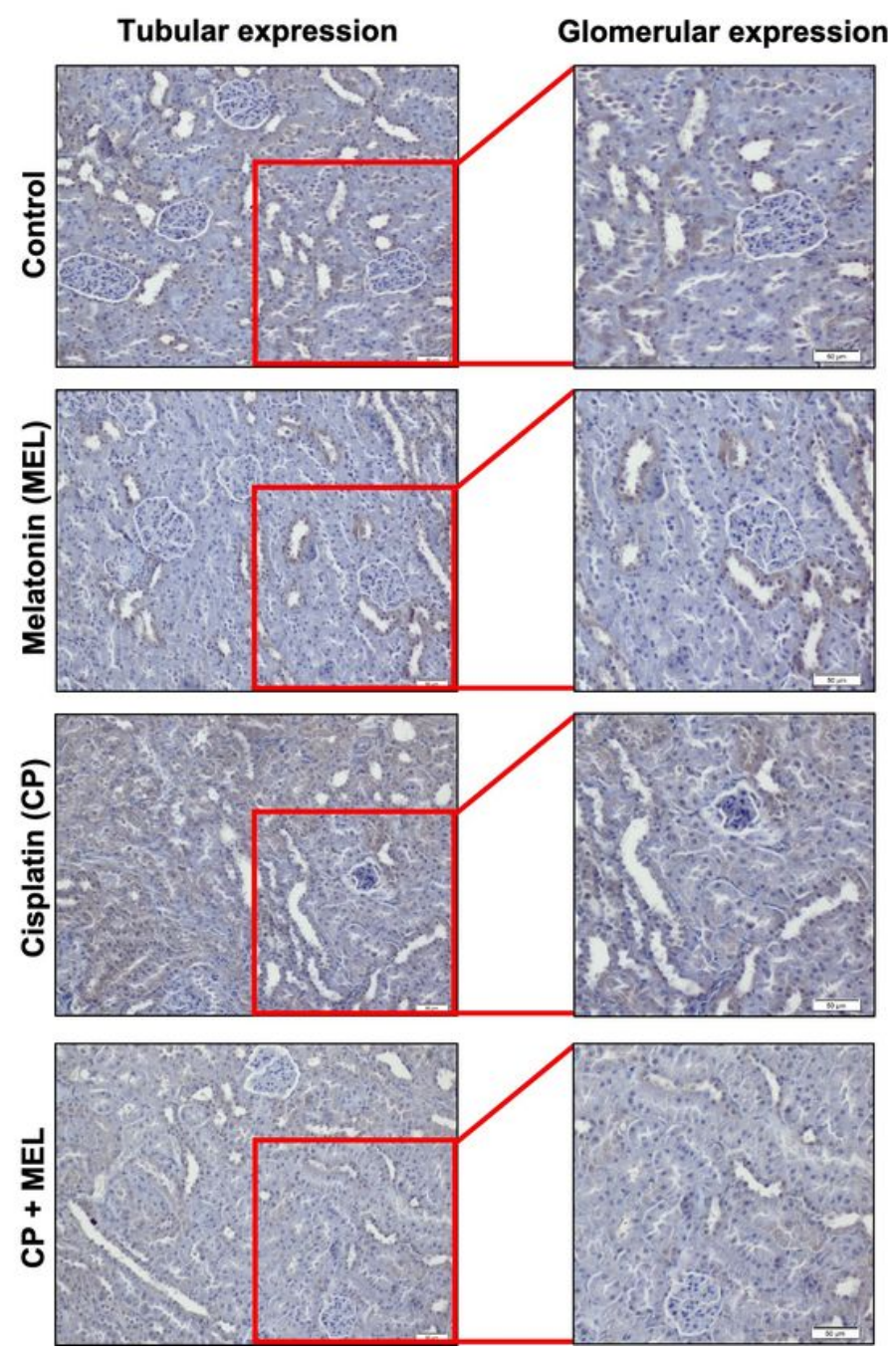

Figure 4

Immunohistochemical staining of HSP70 and HSP90 in kidney sections. Control group $(n=10)$ and MEL group $(n=8)$ showed weak HSP70 immunostaining in both glomerular and tubular region of renal cortex in the kidney tissue; CP group $(n=10)$, HSP70 expressions increased in the glomerular and tubular region. $\mathrm{CP}+\mathrm{MEL}$ group $(\mathrm{n}=10), \mathrm{HSP70}$ expressions decreased when compared with CP group. Similarly, weak HSP90 immunostaining was observed in the kidney sections of Control group $(n=10)$ and MEL group $(n=10)$; CP group ( $n=10)$, HSP90 expression increased in the glomerular and tubular region; CP+MEL $(n=10)$ HSP90 expressions were less compared to the CP group. Scale bar: $50 \mu \mathrm{m}$. Abbreviations: MEL, melatonin; CP, cisplatin; HSP70, heat-shock protein 70; HSP90, heat-shock protein 90. 
Heat-shock protein 47 expression
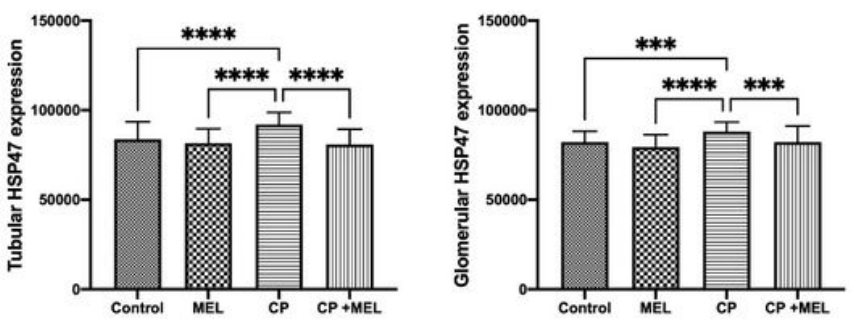

Heat-shock protein 70 expression
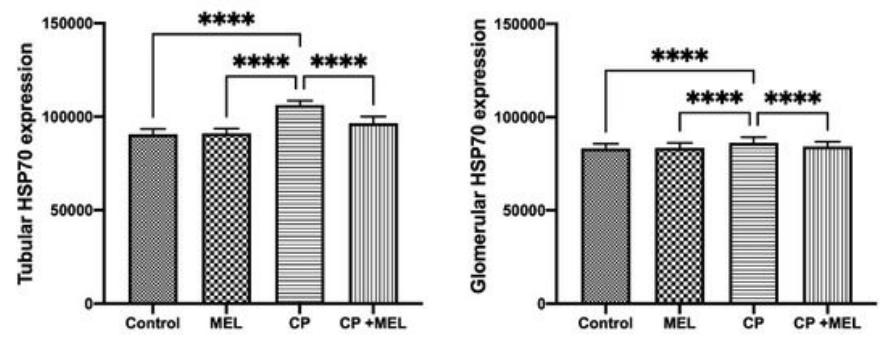

Heat-shock protein 60 expression
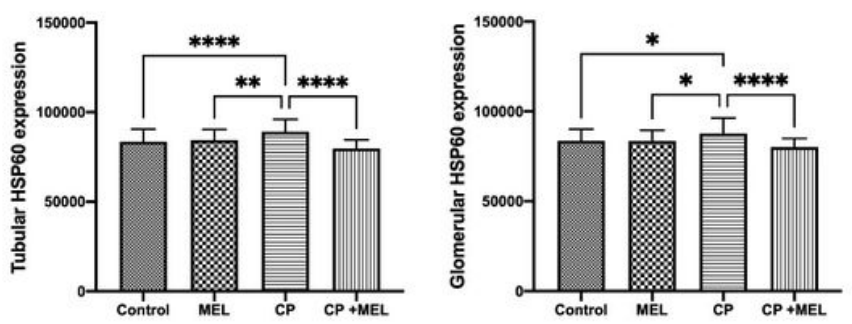

Heat-shock protein 90 expression
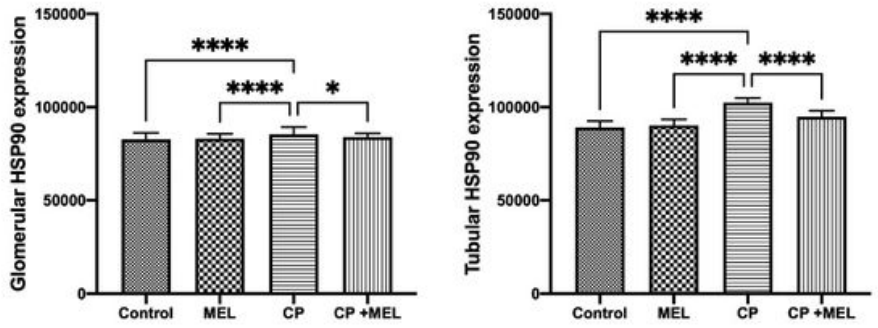

Figure 5

Statistical analysis of the immunoreactivity measurements of the HSP47, HSP60, HSP70 and HSP90 in the glomerular and tubular region of renal cortex of kidney tissue sections of experimental groups. $p$ $<0.05$ was considered as significant. The expression levels of HSP47, HSP60, HSP70 and HSP90 were significantly higher in the CP $(n=10)$ group compared to Control $(n=10)$ and MEL $(n=10)$ groups $(p<0.05)$. Moreover, MEL significantly reduced the increased expression levels of the HSP47, HSP60, HSP70 and HSP90 in the CP+MEL group when compared with the CP group $(p<0.05) .{ }^{*}=p<0.05,{ }^{* *}=p<0.01,{ }^{* * *}=$ $p<0.001, * \star * *=p<0.0001$. Abbreviations: CP, cisplatin; MEL, melatonin; HSP47, heat-shock protein 47; HSP60, heat-shock protein 60; HSP70, heat-shock protein 70; HSP90, heat-shock protein 90.

A

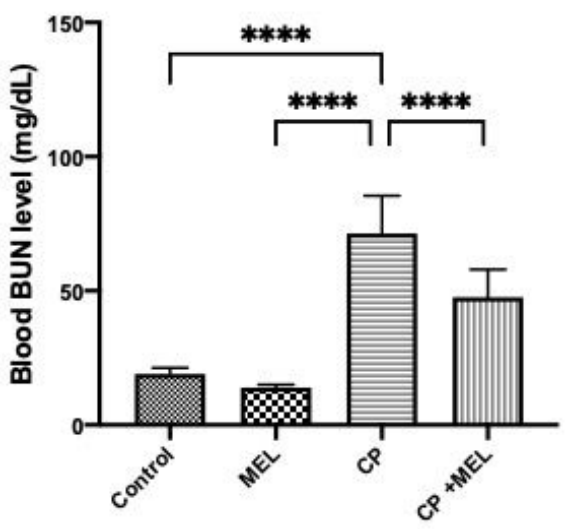

B

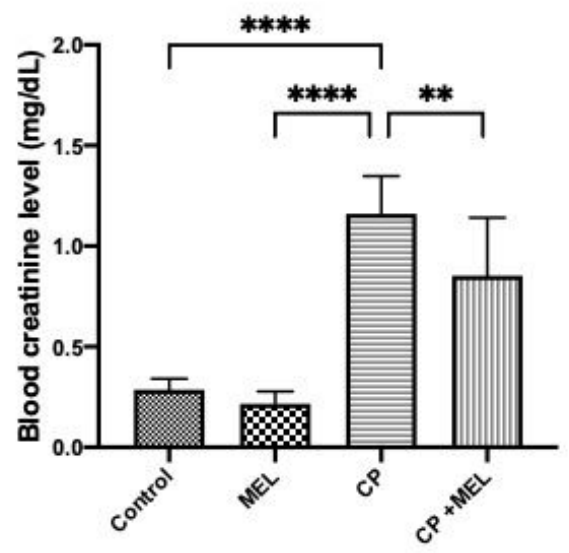

C

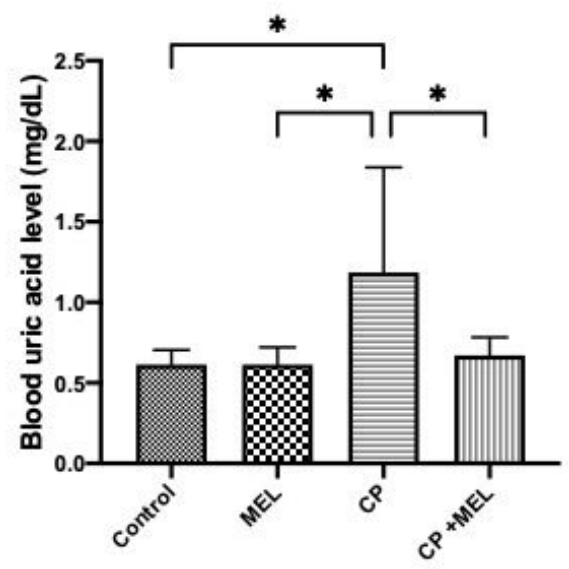




\section{Figure 6}

Measurements of the serum BUN, creatinine and uric acid levels obtained by biochemical assay and statistical analysis of them among experimental groups. $p<0.05$ was considered as significant. CP group $(n=10)$ showed increased serum BUN, creatinine and uric acid levels compared to Control group $(n=10)$ and MEL group $(n=10)$. However, increased serum BUN, creatinine and uric acid levels were observed in CP+MEL group $(n=10)$ compared to CP group. $*=p<0.05, * \star=p<0.01, * \star \star=p<0.001, * \star \star * *=p<0.0001$. Abbreviations: CP, cisplatin; MEL, melatonin; BUN, blood urea nitrogen; HSP47, heat-shock protein 47; HSP60, heat-shock protein 60; HSP70, heat-shock protein 70; HSP90, heat-shock protein 90.

\section{Supplementary Files}

This is a list of supplementary files associated with this preprint. Click to download.

- Biochemical.pzfx

- Histopathology.pzfx

- IHC.pzfx 